\title{
Algorithmische Selektion und Privatheit
}

Aufmerksamkeitssteuerung durch Social Media-Plattformen als Autonomieeingriff?

Stephan Dreyer und Amélie Heldt

Bei der Onlinenutzung kann es passieren, dass unsere Aufmerksamkeit durch andere Akteure im Netz gesteuert wird. Algorithmische Selektionsverfahren bestimmen darüber, welche privaten, halböffentlichen und öffentlichen Informationen wir auf Social Media-Plattformen sehen. Sie bestimmen automatisiert unsere Informationsquellen und damit das Bild, das wir von der Welt und von anderen haben sowie das Bild, das andere sich von uns machen. Während bereits viel dazu geschrieben wurde, welche Relevanz die Beobachtung der individuellen Mediennutzung und die verhaltensorientierte Auswahl von Inhalten durch Plattformen für unser Verständnis von Meinungsbildung hat, macht sich der folgende Beitrag auf die Suche nach den Autonomiebezügen dieser Aufmerksamkeitssteuerung (Abschnitt 2), zeigt eine bislang unterbelichtete grundrechtliche Schutzlücke auf (Abschnitt 3) und schlägt mit Blick auf das lückenschließende Gewährleistungskonzept des Allgemeinen Persönlichkeitsrechts eine neue Grundrechtsausprägung vor: das Recht auf autonomiewahrende Aufmerksamkeitssteuerung (Abschnitt 4).

\section{Neue Aufmerksamkeitsintermediäre und alte Schutzbereiche}

Social Media-Angebote wie soziale Netzwerke, Videoplattformen mit nutzergenerierten Inhalten oder Microblogging-Angebote übernehmen im Internet die Vermittlung der Informations- und Kommunikationsinhalte zwischen den Inhalteanbietern und Nutzenden; eigene Inhalte machen die Plattformen - daher auch der Begriff der "Informationsintermediäre“ ${ }^{1}$ dabei regelmäßig nicht zugänglich. Die Angebote übernehmen angesichts der Fülle der verfügbaren Inhalte Funktionen der Orientierung und Auf-

1 Schulz/Dankert (2016); Stark et al. (2017). 
merksamkeitssteuerung. ${ }^{2}$ Mit Hilfe von Algorithmen filtern, ordnen und priorisieren die Intermediäre Inhalte individuell für die einzelnen Nutzenden. Angesichts ihrer sozialen Verknüpfungen, Vernetzungen und erklärten wie beobachteten Interessen und Vorlieben erhalten alle Nutzenden den individuell errechneten und zusammengestellten ,Stream' bzw. ,Feed vermeintlich relevanter oder interessanter Inhalte. Aus diesem Grund wird den Intermediären in der medienpolitischen Diskussion die potenzielle Relevanz der eingesetzten Verfahren im Hinblick auf die Meinungsbildung, d. h. bezüglich der Formierung von Einstellungen und Haltungen, zugeschrieben (,Relevanzmaschinen $\left.{ }^{6}\right) .{ }^{4}$ Hinsichtlich einer rechtlichen Regulierung von automatisierten Selektionslogiken, etwa zum Schutz vor der Entstehung behaupteter Filterblasen und Echokammern oder zur Wahrung der Integrität von Wahlen, werden beispielsweise die Einführung von Diskriminierungsverboten und die Gewährleistung von Rezeptionsvielfalt auf der Seite der Nutzenden gefordert. ${ }^{5}$ Im Zentrum der Debatten stehen zum Einen die undurchsichtige Auswahl der Inhalte medialer Kommunikation und ihr möglicher, empirisch schwer nachweisbarer Einfluss auf die Meinungsbildung. Zum anderen stellt sich die Frage der Grundrechtsträgerschaft der Intermediäre angesichts ihrer wichtigen Orientierungsfunktion und ihrer Relevanz bei der Konstruktion von Öffentlichkeiten. Beide Thematiken befinden sich im Kern kommunikationsverfassungsrechtlicher Dogmatik und doch ist die grundrechtliche Rückbindung der bisherigen Regulierungsvorschläge systematisch so wenig geklärt wie die Frage eines staatlichen Regulierungsaufgreif- und -auswahlermessens. ${ }^{6}$ Unabhängig davon formt sich in den Rechtswissenschaften eine akademische Phalanx, die angesichts der Ausdifferenzierung der Gesellschaft und der Entgrenzung von Staat und Gesellschaft nach neuen Grundrechtsinnovationen auch mit Blick auf private Grundrechtsträgerschaft ruft. ${ }^{7}$ Gleichzeitig propagiert die Wissenschaft im Hinblick auf das klassische Verständnis von Privatsphäre und ihrer „Verlusterfahrungen"8 die Suche nach neuen Privatheitskonzepten. ${ }^{9}$ Viele sind dem Ruf gefolgt und entsprechend viel

2 Vgl. Dreyer/Schulz (2019a: S. 7).

3 Katzenbach (2017: S. 327).

4 Vgl. Lobigs/Neuberger (2018: S. 68-79).

5 Vgl. Datenethikkommission des Deutschen Bundestages (2019).

6 Vgl. Dreyer/Schulz (2019b: S. 2, 14 f.).

7 Vgl. das gleichnamige Buch von Hornung (2015); Hoffmann-Riem (2016: S. $533 \mathrm{f}$.).

8 Nagenborg (2005: S. 111).

9 Vgl. Albers (2017: S. 21 f.); Stempfhuber (2019: S. 163 f.); Nassehi (2019: 73 f.). 
Literatur gibt es zu den Fragen des Datenschutzes und der Privatheit im Internet und im Kontext der Nutzung sozialer Medien. Im Fokus stehen dabei regelmäßig Rechtsprobleme rund um die Verarbeitung personenbezogener Daten einzelner Personen sowie neue Rechtsfragen, die sich aus der Sammlung und Analyse großer Datenmengen, im Sinne von ,Big Data', ergeben. ${ }^{10}$ Die Konsequenzen aus der algorithmischen Selektion von Informationen Dritter für die einzelnen rezipierenden Nutzenden sind dabei meist nicht zentraler Untersuchungsgegenstand.

Diesen Umstand nimmt der Beitrag zum Anlass, die bislang kaum untersuchten Privatheitsbezüge der Aufmerksamkeitssteuerung im Internet in den Fokus zu rücken und sie in die bestehenden grundrechtlichen Schutzbereiche und neueren Konzepte von Privatheit einzuordnen. Es soll geklärt werden, inwieweit eine grundrechtliche Schutzbereichslücke für Aspekte der Aufmerksamkeitssteuerung bei algorithmischer Selektion besteht und ob sich dabei eine möglicherweise neue Schutzbereichsausprägung des Allgemeinen Persönlichkeitsrechts aus Privatheitsüberlegungen herleiten ließe.

\section{Algorithmische Selektion und die kommunikative Dimension von Privatheit}

Zunächst soll eine Begriffsbestimmung dessen vorgenommen werden, was ,algorithmische Selektion' umfasst und auf welchen Ebenen diese Selektion zu einer Aufmerksamkeitssteuerung führt.

\subsection{Zur Begrifflichkeit algorithmischer Selektion}

Unter algorithmischer Selektion werden hier algorithmische Systeme verstanden, die auf Grundlage digitaler Daten arbeiten, regelmäßig das individuelle Nutzendenverhalten in aggregierter Form als Input einbeziehen, auf dieser Grundlage Auswahlen, Priorisierungen und Rankings, Klassifikationen oder statistische Assoziationen vornehmen und auf dieser Grundlage Medien- oder Kommunikationsinhalte als Output selektieren. ${ }^{11}$ Bei der Einbeziehung von Nutzendenverhalten in die Selektion kann zwischen drei Formen unterschieden werden: So kann a) eine bewusste unmittelbare Beteiligung an der algorithmischen Selektion erfolgen, etwa im

10 Vgl. die Beiträge in Hoffmann-Riem (2018).

11 Vgl. Schmidt et al. (2017: S. 20); Napoli (2014). 
Rahmen der aktiven nutzungsseitigen Offenbarung von Interessen, Vorlieben oder sozialen Verbindungen (z. B. über Abos, Followings, Freundeslisten, Bekanntennetze, Angabe von Interessen oder Hobbies). Ebenfalls können b) unbewusst offenbarte Faktoren oder mittelbar durch Auswertungen entstandene Inferenzdaten (z. B. beobachtete Verhaltensdaten, Metadaten, aus dem Bekannten-/Followingkreis erfolgte Deduktionen) in die Selektionsverfahren einbezogen werden. Daneben können c) stets Auswahl- und Priorisierungsverfahren Anwendung finden, die keine personenspezifische Beteiligung der Nutzenden an der algorithmischen Selektion erfordern, sondern die relevante Inhalte nach dem Verhalten anderer Nutzender oder über inhaltsbezogene Klassifikationsmöglichkeiten selektieren (z. B. content oder context based filtering). ${ }^{12}$ Alle drei Formen derartiger algorithmischer Selektionsverfahren finden sich bei sozialen Netzwerken, Foto- und Videoplattformen für nutzungsseitig generierte Inhalte sowie Microblogging-Plattformen.

Insgesamt werden durch diese automatisierten Auswahlverfahren Informationsflüsse strukturiert, die sich auf der Seite der Nutzenden als Aufmerksamkeitssteuerung ${ }^{13}$ darstellen: Die Selektionsverfahren bestimmen, welche publizistischen Informationen, welche Informationen über welche Freunde, Bekannte oder andere Profile Nutzende erhalten und welche Informationen über diese Nutzende wiederum deren Freunde, Bekannte und Follower angezeigt bekommen.

\subsection{Privatheits- und Autonomiebezüge der aufmerksamkeitslenkenden Strukturierung von Online-Inhalten}

Wissenschaftliche Arbeiten haben sich umfassend mit Privatsphäre und Privatheit in digitalen Kommunikationsräumen auseinandergesetzt. Der Diskurs ist zu großen Teilen von dem Umgang mit personenbezogenen Daten und der Beobachtbarkeit bzw. Profilierung der Nutzenden durch digitale Plattformen geprägt. Hier stehen die Beobachtbarkeit und die Datenpreisgabe aus einer klassisch individualistischen Perspektive im Fokus der Betrachtungen. Daneben gibt es Zugänge zum Thema, die soziale und kollektive Dimensionen von Privatheit erkannt und anerkannt haben, ${ }^{14}$ während die spezifisch medienvermittelten Dimensionen von Privatheit

12 Schmidt et al. (2018: S. 523 f.).

13 Zum Begriff Mengden (2018: S. 150).

14 Vgl. Rössler (2001: S. 234-251); Rössler (2018); Gusy, KritV 2015, 430. 
bislang seltener herausgearbeitet wurden. ${ }^{15}$ Doch selbst bei diesen Ausführungen kommt die Komponente, dass Social Media-Nutzung stets auch eine aktive kommunikative Grundrechtsausübung darstellt, zu kurz. Folgt man aber dem Denkansatz, dass Grundrechteschutz in erster Linie aktiver Ausübungsschutz ist, ${ }^{16}$ kommt es bei der Social Media-Nutzung auf die Handlungsformen der Nutzenden an. Nach Schmidt weist die Nutzung hier im Wesentlichen drei Handlungskomponenten auf: das Informationsmanagement, das Beziehungsmanagement und das Identitätsmanagement. ${ }^{17}$

Im Folgenden soll gezeigt werden, dass die algorithmische Selektion das heißt, die spezifische Filterentscheidung, ob ein bestimmter Inhalt gezeigt und wie dieser Inhalt vom System priorisiert wird - in allen drei Dimensionen jeweils deutliche Bezüge zu Privatheitsaspekten aufweist.

\subsubsection{Informationsdimension}

Mit Blick auf informationsbezogene Social Media-Nutzungspraktiken sollen die Plattformen die unterschiedlichen Informationsbedürfnisse und -interessen der Nutzenden befriedigen, seien es geäußerte oder ungeäußerte, individuelle oder gesellschaftsbezogene Interessen. Die Selektion der Inhalte durch die Plattform hat insoweit Bedeutung für den individuellen Zugang zu Informationen und - damit verschränkt - auch für die individuelle Wahrnehmung der Welt durch Nutzende. Die Selektion und Priorisierung bestimmter Themen, Entwicklungen und Vorkommnisse entspricht dem klassischen ,Agenda Setting': Die Auswahl von spezifischen Themen und die Nicht-Auswahl anderer können Nutzende in ihrer Wahrnehmung der jeweils relevanten gesellschaftlichen Diskurse beeinflussen. Daneben hat die Selektion Relevanz für die Vermittlung von Informationen über und die Repräsentation von Meinungen und Haltungen zu einem bestimmten Thema (,Meinungsklima', ,Framing ${ }^{6}$ ). Insgesamt sind die Selektionsverfahren in der Lage, auf Seiten der Nutzenden ein Bild von der Welt und der eigenen Umwelt und damit eine individuelle (und ggf.

15 Vgl. Becker (2017); Becker/Seubert, DuD 2016, 73.

16 Vgl. Staben (2016: S. 103). In erster Linie geht es bei Grundrechten um den Schutz ihrer Ausübung; die Gewährleistung der negativen Komponente eines Grundrechts - z. B. das Recht, sich nicht zu äußern, sich nicht zu versammeln, nicht an eine Religion zu glauben - spielt in der Praxis eine untergeordnete Rolle.

17 Vgl. Schmidt (2006); Zerfaß et al. (2008: S. 24); Schmidt (2017). 
verzerrte) Realität zu konstruieren. Nur ein Teil dieses Output-Weltbildes beruht auf dem Input in Form bewusst geäußerter Informationsbedürfnisse der Nutzenden gegenüber Intermediären, anders als etwa bei selbst zusammengestellten Informationsrepertoires und aktiv aufgerufenen Informationsangeboten ohne vermittelnde Zwischenleistung; bei Informationsintermediären fügen algorithmische Filter-, Sortierungs- und Priorisierungsverfahren weitere Inhalte hinzu oder blenden andere aus der Wahrnehmung der Nutzenden aus.

Diese durch Intermediäre extern gesteuerten Meinungs- und Willensbildungsbezüge sind vielleicht der relevanteste Aspekt der Selektion: Wenn die Realitätskonstruktion fremdbestimmt ist, erscheint dies als Gegenstück zur wahrnehmungsbezogenen Selbstbestimmung - eine autonome Wahrnehmung der Welt wird herausgefordert oder gar ersetzt durch Entscheidungen eines Algorithmus. ${ }^{18}$ Unabhängig davon, ob der Algorithmus und die Plattformbetreiber gutwillig handeln: Der Autonomiebezug der Selektion ist da, und, soweit die innere Meinungs- und Willensbildung in Form von Gedankenbewegungen betroffen ist, wird damit auch ein Aspekt von (dezisionaler) Privatheit berührt. ${ }^{19}$

Ein weiterer Autonomieaspekt kann darin zu sehen sein, dass sich ein durch die Informationsauswahl wahrgenommenes vermeintliches Meinungsklima ggf. auf die eigene Kommunikationsaktivität der Nutzenden auswirkt. ${ }^{20}$ Wenn die wahrgenommene Ansicht einer vermeintlichen Mehrheit oder der Umgang dieser Mehrheit mit Andersdenkenden jemanden dazu bringt, sich nicht oder in anderer Weise zu äußern, dann ist neben der Ausübung der Meinungsäußerungsfreiheit auch die Autonomie der Einzelnen betroffen.

Ein dritter Privatheits- und Autonomiebezug entsteht durch die Beobachtung und Auswertung des Nutzungsverhaltens durch die Plattformbetreiber. Bereits durch Unsicherheit über die Art und Weise der Beobachtbarkeit und der potentiellen Auswertung sowie der damit möglichen Dekontextualisierung von Wissen über Nutzende können sich diese zur Anpassung ihres Nutzungsverhaltens veranlasst sehen. ${ }^{21}$ Auch derartige Hem-

18 Unstrittig bleibt, dass die Wahrnehmung der Welt auch bei klassischen Medien eine eingeschränkte und vorselektierte ist. Der kategoriale Unterschied ist, dass die klassische Medienselektion auf Grundlage journalistischer Logiken und Selbstverständnisse erfolgt.

19 Vgl. Albers (2017: S. 24).

20 Vgl. Petersen et al. (2017).

21 Vgl. Mühlichen (2018: S. 34 f.); in der Praxis konnten diese Effekte in Experimenten jüngst nicht nachgewiesen werden, vgl. Braun et al. (2018). 
mungen weisen klare Autonomiebezüge auf. Ähnlich relevant können von Auswertungsverfahren erkannte, aber nicht bewusst geäußerte Informationsbedürfnisse oder Interessen sein: Durch prädiktive Verfahren können ggf. Persönlichkeitsmerkmale oder individuelle Vorlieben ,entdeckt ${ }^{\star}$ werden, ohne dass die Nutzende dies wünscht. Von den berührten Menschenwürdeaspekten abgesehen, ${ }^{22}$ kann die Angst vor derartigen mathematischstatistischen Entdeckungen, seien sie tatsächlich oder eben nur vermeintlich, ebenfalls zu Verhaltensanpassungen führen, und so Privatheits- und Autonomiebezüge aufweisen. ${ }^{23}$

\subsubsection{Beziehungsdimension}

Auf der Ebene des Beziehungsmanagements ist die algorithmisierte Aufmerksamkeitssteuerung auf Social Media-Plattformen für die Sichtbarkeit von persönlichen Informationen und Kommunikationen Dritter entscheidend. Damit hat sie Relevanz für die Entwicklung von sozialen Beziehungen: Die Häufigkeit und Vollständigkeit der zugänglich gemachten Informationen über einzelne Freunde oder Bekannte hat Einfluss darauf, wie nah Nutzende sich ihnen und ihren Leben fühlen. ${ }^{24}$ Bekannte, die bei der Selektion depriorisiert werden, können aus diesem Näheverhältnis herausfallen oder mitunter in Vergessenheit geraten. Die Auswahl, ob und welche Informationen von welchen Freunden und Bekannten den Nutzenden verfügbar gemacht werden, erscheint so als externe Bekräftigung, Schwächung oder jedenfalls Steuerung von ,Freundschaft ${ }^{6}$ und sozialen Beziehungen zwischen den Nutzenden der Plattform. ${ }^{25}$ Angesichts der Bedeutsamkeit des Freundes- und Bekanntenkreises als Sozialisationsinstanz weist dieser Aspekt eine hohe Relevanz für die eigene Persönlichkeitsentwicklung und -entfaltung auf. Gusy ${ }^{26}$, Trepte ${ }^{27}$ und Rössler ${ }^{28}$ haben die soziale Dimension von Privatheit herausgearbeitet und beschrieben, wie unterschiedliche Privatheiten innerhalb von sozialen Beziehungen und in Abgrenzung $\mathrm{zu}$ anderen Beziehungen kommunikativ verhandelt werden.

22 Die einzelne Person erscheint hier nur noch als berechenbares und berechnetes Artefakt; vgl. Dreyer (2018).

23 Vgl. Solove (2004: S. 35).

24 Vgl. Donath (2007); Tufekci (2008); Thelwall/Wilkinson (2010).

25 Vgl. Ellison et al. (2014); Tong/Walther (2011).

26 Vgl. Gusy, KritV 2015, 41.

27 Vgl. Trepte (2016).

28 Vgl. Rössler (2001: S. 201-254); Rössler (2018). 
Üben Dritte Einfluss auf diese Beziehungen und deren Ausmaß von Nähe und Distanz aus, so erscheint diese Steuerung auch als Handlung mit Privatheitsbezug.

\subsubsection{Identitätsdimension}

Die Einflussnahme von Social Media-Plattformen auf die Verfügbarmachung von Kommunikationsinhalten ergibt sich auch in die andere Richtung: Wie werden die von mir preisgegebenen Informationen bei anderen Nutzenden sichtbar? In dieser Dimension können Intermediäre Relevanz für das individuelle Identitätsmanagement entfalten. Die Aufmerksamkeitssteuerung weist auf dieser Ebene Berührungspunkte zu der Schaffung und ggf. Bestätigung eines bestimmten Selbstbildes auf der Seite von Dritten auf. ${ }^{29}$ Durch algorithmische Selektionslogiken haben die Nutzenden selbst keinen Einfluss (mehr) darauf, welche Informationen über sie bei Freunden, Bekannten und Followern ankommen. Einige der Empfangenden erhalten ggf. nur einen Ausschnitt der Mitteilungen, andere vielleicht gar keine. Insbesondere bei der selektiven Auswahl der zugänglich gemachten Informationen können sich so Zerrbilder über die Nutzenden auf der Seite der Rezipierenden bilden. So hat die algorithmische Selektion unmittelbaren Einfluss auf die Möglichkeiten der informationellen Selbstdarstellung der Einzelnen, d. h. auf die Darstellung der eigenen Person gegenüber Dritten. Anders formuliert haben die Selektionslogiken der Social Media-Plattformen abträgliche Potenziale für die Schaffung und ggf. Bestätigung eines bestimmten - erwünschten - Fremdbildes.

Das bewusste Management der Darstellung der eigenen Person gegenüber Dritten nennt Schmidt ,Identitätsmanagement ${ }^{630}$. Steinmüller et al. führen mit Blick auf handlungsfreiheitsrelevante Aspekte den Begriff „informationelle Selbstdarstellung" 31 ein und zeigen mit Blick auf den Aspekt der eigenen Steuerung von Fremdbildern unmittelbar wirkende Autonomiebezüge auf. Auch mittelbare Bezüge lassen sich hier erkennen: So kann die (ggf. verzerrte) Wahrnehmung der eigenen Person bei Dritten zu Rückwirkungen auf die eigene Entwicklung und Entfaltung führen, etwa

29 Vgl. Krämer/Haferkamp (2011); Bazarova et al. (2012).

30 Vgl. Schmidt (2006); Schmidt (2017).

31 Steinmüller et al. (1971: S. 87). 
durch Feedback oder die von der Eigenwahrnehmung abweichende Fremderwartung. ${ }^{32}$

\section{Grundrechtsmapping}

Die Betrachtung der möglichen Wirkungen algorithmischer Selektion auf Social Media-Plattformen hat gezeigt, dass diese auf ganz unterschiedlichen Ebenen Relevanz für Privatheit und Autonomie Einzelner erlangen können. Die Verfahren zur Filterung und Sortierung von Informationsund Kommunikationsinhalten können Auswirkungen auf die Informationsauswahl haben, sie können Beziehungen festigen oder schwächen und sie weisen Einflusspotenziale im Hinblick auf das eigene Identitätsmanagement auf. Fraglich bleibt, inwieweit diese Aspekte bereits durch bestehende grundrechtliche Gewährleistungsgehalte abgedeckt sein können - dann nämlich würde sich ein weiteres Nachdenken über neuartige Schutzbereichsausprägungen und Anknüpfungspunkte legislativer Rechtfertigungen erübrigen. Ein Grundrechtsmapping ist also für die darauffolgenden Überlegungen nötig und soll im Folgenden kurz aufgefächert werden.

\subsection{Autonomie als Zielwert der allgemeinen Handlungsfreiheit}

Autonomie, d. h. die Möglichkeit zur ungestörten, selbstbestimmten Entwicklung, Entfaltung und Wahrung der eigenen Individualität, steht im Zentrum der allgemeinen Handlungsfreiheit, wie sie Art. 2 Abs. 1 GG gewährleistet. ${ }^{33}$ Das Verständnis von Autonomie als normativer Zielwert ist dabei umfassend entwicklungs-, entfaltungs- und bewahrungsbezogen. Den Staat trifft hier ein Gewährleistungsauftrag, der auf die Herstellung eines Rechtsrahmens zielt, sodass die private Autonomie Einzelner möglichst umfassend erhalten bleibt. ${ }^{34}$ Davon umfasst ist auch der Respekt vor der geschützten Persönlichkeitsentfaltung durch den Staat wie durch Dritte. Im Folgenden werden Grundrechtsbereiche unterschieden, die als Aus-

32 Vgl. Smith (2017); zum Konzept der digitalen persona siehe Clarke (1994); Clarke (2014); zu den Autonomiebezügen dieses Konzepts siehe Pasquale/Cockfield (2018: S. $861 \mathrm{f.})$.

33 Vgl. Di Fabio (87. EL, März 2019). In: Maunz/Dürig, GG, Art. 2 Abs. 1, Rn. 131.

34 Wenn auch dem Gesetzgeber dabei ein großer Einschätzungsspielraum bleibt, vgl. Di Fabio (87. EL, März 2019). In: Maunz/Dürig, GG, Art. 2 Abs. 1, Rn. 61, Rn. 135 f. 
prägungen des Rechts auf Persönlichkeitsentwicklung und -entfaltung erscheinen (oder jedenfalls Bezüge dazu haben), und solchen, die spezifische informations- und kommunikationsbezogene Aspekte aufweisen.

\subsection{Persönlichkeitsrechtliche Ausprägungen}

\subsubsection{Recht auf Privatsphäre}

Privatheit oder Privatsphäre sind Begriffe, die das Grundgesetz nicht kennt. Vielmehr wurde ein unantastbarer Bereich der privaten Lebensgestaltung ${ }^{35}$ vom Bundesverfassungsgericht früh definiert und später in ein den Schutz der Privatsphäre als Untergewährleistung eines Allgemeinen Persönlichkeitsrechts aus Art. 2 Abs. 1 in Verbindung mit Art. 1 Abs. 1 GG weiterentwickelt. ${ }^{36}$ Nach Sicht des Gerichts umfasst der Schutz der Privatsphäre der Einzelnen einen räumlich und thematisch bestimmten Bereich, der grundsätzlich frei von unerwünschter Einsichtnahme bleiben soll. ${ }^{37}$ Ein relevanter Teilaspekt der Gewährleistung ist dabei das Recht, Beziehungen zu anderen Personen zu knüpfen und zu pflegen. ${ }^{38}$ Während es beim Recht auf Privatsphäre um die Anwendung auf den engeren persönlichen Lebensbereich geht, betrifft die algorithmische Selektion (wie oben gezeigt) vor allem Informationen, die Nutzende oder Dritte bewusst zugänglich gemacht haben.

Das Recht auf Privatsphäre erscheint wegen dieses inhärenten Widerspruchs insoweit (nur) angesichts von Selektionsentscheidungen tangiert, die (a) auf Grundlage geschlussfolgerter Annahmen und Profilierungen vorgenommen werden, die Nutzende selbst nicht öffentlich gemacht haben, dem Bereich der höchstpersönlichen Lebenssphäre zuzuordnen sind und auf die sie kaum oder keinen unmittelbaren Einfluss haben (Inferenz), und (b) die faktische Verhinderung des Führens sozialer Beziehungen

35 Vgl. BVerfG Beschluss vom 31.01.1973 - 2 BvR 454/71, BVerfGE 34, 238 (247 f.).

36 Vgl. BVerfG Beschluss vom 24.02.1971 - 1 BvR 435/68, BVerfGE 30, 174; BVerfG Beschluss vom 03.06.1980 - 1 BvR 185/77, BVerfGE 54, 148; siehe dazu Nettesheim, VVDStRL 2011, 7.

37 Vgl. BVerfG Beschluss vom 15.01.1970 - 1 BvR 13/68, BVerfGE 27, 344 (350 ff.); BVerfG Beschluss vom 24.05.1977 - 2 BvR 988/75, BVerfGE 44, 353 (372f.); BVerfG Beschluss vom 26.04.1994 - 1 BvR 1689/88, BVerfGE 90, 255 (260); BVerfG Urteil vom 15.12.1999 - 1 BvR 653/96, BVerfGE 101, 361 (382 f.). Vgl. EGMR Urteil vom 13.02.2003 - 42326/98, NJW 2003, 2145, Rn. 29. 
durch systematische Ausblendung von einzelnen Freundinnen und Bekannten.

\subsubsection{Recht am eigenen Wort und Bild}

Das Recht am eigenen Wort und Bild als weitere Untergewährleistung des Allgemeinen Persönlichkeitsrechts besteht im Kern aus dem Recht, die Darstellung der eigenen Person gegenüber Dritten grundsätzlich selbst zu bestimmen. ${ }^{39}$ Die Möglichkeit, von Anderen so wahrgenommen zu werden, wie die Grundrechtsträgerin sich selbst sieht, umfasst das Recht aber gerade nicht. ${ }^{40}$ Insoweit begründet das Recht im traditionellen Verständnis auch keinen Anspruch auf eine bestimmte Auswahl von Bildern oder Kommunikationen durch die Nutzenden. Anwendung findet das Recht am eigenen Wort bzw. Bild dagegen vor allem auf nicht gewollte Veröffentlichungen: Die Ausprägung des Allgemeinen Persönlichkeitsrechts schützt vor Vertrauensbrüchen. Bei der algorithmischen Selektion auf Social Media-Plattformen geht es allerdings regelmäßig um Informationen, die von anderen gesehen werden sollen. Das Recht am eigenen Bild oder Wort in dem hier verstandenen Sinne wäre durch Selektionsverfahren insoweit nicht tangiert. Ein Beispiel, bei dem das Recht am eigenen Bild höchstens berührt sein könnte, wären Sachverhalte, in denen durch die Selektion einer Plattform das Bild einer Nutzerin missbräuchlich dekontextualisiert würde, etwa bei der Nutzung zu werblichen Zwecken.

\subsubsection{Informationelle Selbstbestimmung}

Auf den ersten Blick besonders einschlägig erscheint das Recht auf informationelle Selbstbestimmung, das einen weiteren Unterbereich des Allgemeinen Persönlichkeitsrechts darstellt. Das Recht besteht im Kern aus dem Selbstbestimmungsrecht der Einzelnen darüber, wann und innerhalb welcher Grenzen persönliche Lebenssachverhalte offenbart werden sollen. ${ }^{41}$

39 Vgl. BVerfG Urteil vom 15.12.1999 - 1 BvR 653/96, BVerfGE 101, 361 (381); BVerfG Urteil vom 17.02.1998 - 1 BvF 1 /91, BVerfGE 97, 228 (268 f.).

40 Vgl. BVerfG Beschluss vom 10.11.1998 - 1 BvR 1531/96, BVerfGE 99, 185 (194); BVerfG Urteil vom 15.12.1999 - 1 BvR 653/96, BVerfGE 101, 361 (380).

41 Vgl. BVerfG Urteil vom 15.02.2006 - 1 BvR 357/05, BVerfGE 115, 11 (183 ff.); BVerfG Urteil vom 15.12.1999 - 1 BvR 653/96, BVerfGE101, 361 (383); BVerfG 
Das informationelle Selbstbestimmungsrecht als negatives Freiheitsrecht ${ }^{42}$ schützt die Entscheidungsmacht darüber, wer was wann über einen selbst weiß. ${ }^{43}$ Personenbezogene Informationen, die selbst und bewusst veröffentlicht wurden, sind grundsätzlich nicht von diesem Recht umfasst. Auch ergibt sich daraus kein positiver Rechtsanspruch auf eine bestimmte Darstellung der eigenen Person gegenüber Dritten, etwa in Form eines Anspruchs auf Aufmerksamkeit (s. oben). Das Recht auf informationelle Selbstbestimmung kann so auch keine Ansprüche erzeugen, die auf Fragen des Outputs algorithmischer Selektion gerichtet sind.

Datenschutzrechtliche Auskunftsansprüche befassen sich mit den auf Plattformseite vorliegenden Daten zu einer bestimmten Person, nicht aber mit der Selektionskonsequenz auf Grundlage dieser Daten. Ebenfalls geht das Recht fehl im Hinblick auf den Schutz vor ggf. vielfaltseinengenden Selektionsverfahren, die Informationen betreffen, die an einen bestimmten Nutzenden gerichtet sind oder diesem vorenthalten werden. Letztlich hilft das Recht auf informationelle Selbstbestimmung nicht bei dem Schutz vor dem Ausblenden oder Depriorisieren relevanter Informationen aus dem ,Freundeskreis‘.

Etwas Anderes kann für Sachverhalte gelten, bei denen der Aspekt der Beobachtung bzw. Beobachtbarkeit eine Rolle spielt: So kann die Aggregation und Auswertung unbewusst erzeugter Daten und die Deduktion neuer (personenbezogener) Informationen die Grenze zur Privat- und ggf. Intimsphäre überschreiten, etwa bei höchstpersönlichen Eigenschaften, Interessen und Vorlieben. ${ }^{44}$ Diese Formen der Beobachtung und die Deduktion selbst sind dabei durchaus von dem Schutzbereich des informationellen Selbstbestimmungsrechts umfasst ${ }^{45}$ - nicht aber die daraus folgenden Konsequenzen für die Aufmerksamkeitssteuerung.

Beschluss vom 10.11.1998 - 1 BvR 1531/96, BVerfGE 99, 185 (193); BVerfG Beschluss vom 02.05.2006 - 1 BvR 507/01, NJW 2006, 2836 ff.

42 Vgl. zu objektiv-rechtlichen Komponenten Ladeur, DÖV 2009, 45 (54f.).

43 Vgl. BVerfG Urteil vom 15.12.1983 - 1 BvR 209, 269, 362, 420, 440, 484/83, BVerfGE 65, 1 (43).

44 Vgl. die Studie von Kosinski/Wang (2018), bei der ein Algorithmus anhand von Fotoaufnahmen einzelner Personen die Wahrscheinlichkeit ihrer Homosexualität berechnet hat. Dass die Untersuchung kritisiert wurde, weil die neuronalen Netzwerke die sexuelle Orientierung der Testpersonen ggf. nicht anhand der Gesichtszüge, sondern anhand modischer Merkmale berechnet hat, spielt für das Argument hier keine Rolle.

45 Vgl. das Urteil des OLG Köln zum Umfang des Auskunftsanspruchs bei auf Seiten der verantwortlichen Stelle hinzugefügten Daten (hier: Telefonnotizen), OLG Köln, Urteil v. 26.07.2019 - 20 U 75/18. 


\subsubsection{Recht auf Vertraulichkeit und Integrität informationstechnischer Systeme}

Das relativ junge Recht auf Vertraulichkeit und Integrität informationstechnischer Systeme, das das Bundesverfassungsgericht 2008 erstmals formuliert hat, ${ }^{46}$ und welches seitdem unter dem Begriff ,IT-Grundrecht' in der wissenschaftlichen Diskussion Fuß gefasst hat, umfasst im Kern den Schutz vor technischen Zugriffsmöglichkeiten von Dritten auf IT-Systeme der Grundrechtstragenden, die zum Ausspähen oder zur Manipulation von auf diesen Systemen vorhandenen Daten genutzt werden ${ }^{47}$; später stellte das Gericht klar, dass davon auch IT-Systeme außerhalb der privaten Wohnung umfasst sind, etwa Daten in sozialen Netzwerken oder der sog. Cloud. ${ }^{48}$ Das als Auffanggrundrecht entwickelte ,IT-Grundrecht' zielt damit in erster Linie auf Fälle $\mathrm{ab}$, in denen Einzelne vor dem Auslesen vertraulicher Informationen durch (staatliche) heimliche Infiltration von ITSystemen geschützt werden sollen. Angesichts der Kontexte der algorithmischen Selektion auf Plattformen erscheint die Auffangfunktion des ,IT-Grundrechts' ins Leere zu greifen: Die selektierten Daten sind nicht vertraulich, und die Selektion erfolgt zwar oft undurchsichtig, aber nicht heimlich. ${ }^{4}$

\subsubsection{Spezifische Privatheitsgrundrechte: Unverletzlichkeit der Wohnung und Fernmeldegeheimnis}

Das Grundgesetz kennt mit der Unverletzlichkeit der Wohnung (Art. 13 GG) und dem Fernmeldegeheimnis (Art.10 GG) zwei spezifische Grundrechtsverbürgungen, die sich dem Schutz bestimmter Aspekte der Privatsphäre widmen. Beide sehen den Schutz vor staatlichen Eingriffen in die Privatsphäre als zentral an. Dabei umfasst der Schutzbereich von Art. 13 GG den real-räumlichen Bereich der eigenen Wohnung und gerade nicht einen virtuell-informationstechnischen Bereich freier Persönlichkeitsentfaltung. ${ }^{50}$ Diese physische Begrenzung des Schutzbereichs war eine

46 BVerfG Urteil vom 27.02.2008 - 1 BvR 370, 595/07, BVerfGE 120, 274.

47 BVerfG Urteil vom 27.02.2008 - 1 BvR 370, 595/07, BVerfGE 120, 274 (314).

48 BVerfG Urteil vom 20.04.2016 - 1 BvR 966, 1140/09, BVerfGE 141, 220 (303).

49 Inwieweit das schlichte Nicht-Wissen derartiger Verarbeitungspraktiken auf Seite der Nutzenden einer Heimlichkeit aus einer verfassungsrechtlichen Wirkungssicht gleichkommt, ist in der Rechtswissenschaft noch nicht vertieft diskutiert.

50 Vgl. BVerfG Urteil vom 27.02.2008 - 1 BvR 370, 595/07, BVerfGE 120, 274 (312 ff.). 
der Ursachen für die Entwicklung des ,IT-Grundrechts“ (s. oben). Auch der vom Fernmeldegeheimnis anvisierte Schutz vor staatlicher Überwachung von telekommunikativ vermittelter Individualkommunikation passt nicht für halböffentliche und öffentliche Informations- und Kommunikationshandlungen auf sozialen Netzwerkplattformen. ${ }^{51}$ Als in erster Linie abwehrrechtlich konzipiertes Grundrecht kann aus Art. 10 GG auch kein Anspruch auf eine etwaige positive Berücksichtigung der Übertragung einzelner Kommunikationsinhalte in Fernmeldeinfrastrukturen erwachsen.

\subsection{Informations- und kommunikationsbezogene Ausprägungen}

Klar ist, dass die Nutzung von Social Media-Plattformen stets auch Kommunikationsgrundrechte berührt: Die Beschaffung und Rezeption von Informationen über die Welt und Dritte, die Kommunikation mit Freunden und Bekannten und die Darstellung der eigenen Person auf und über entsprechende Plattformen erfolgt technikimmanent stets medial vermittelt.

\subsubsection{Informationsfreiheit}

Das Grundrecht auf Informationsfreiheit in Art. 5 Abs. 1 S. 1 GG ist auf die Möglichkeit einer unbeeinträchtigten Meinungs- und Willensbildung gerichtet: Nur, wenn die Einzelne sich aus allgemein zugänglichen Quellen ungehindert informieren kann, ist eine aktive Teilnahme am demokratischen Entscheidungsfindungsprozess möglich. ${ }^{52}$ Nur informierte Personen können sich zu einzelnen Sachfragen eine eigene Meinung bilden und diese im Bedarfsfall auch äußern.

Allerdings bezieht sich die Informationsfreiheit lediglich auf den $\mathrm{Zu}$ gang zu einmal eröffneten Quellen. ${ }^{53}$ Algorithmische Selektionsverfahren löschen aber keine verfügbaren Informationsquellen und stellen regelmäBig auch keine Behinderung des Informationszugangs dar, sondern steuern die Aufmerksamkeit der Einzelnen ggf. nicht auf diese Information.

51 Vgl. BVerfG Beschluss vom 24.01.2012 - 1 BvR 1299/05, BVerfGE 130, 151 (179f.); Schoch, Jura 2011, 194.

52 Vgl. BVerfG Beschluss vom 03.10.1969 - 1 BvR 46/65, BVerfGE 27, 71 (81f.).

53 Vgl. Grabenwarter (87. EL März 2019). In: Maunz/Dürig, GG, Art. 5 Abs. 1, Abs. 2, Rn. 1006 ff. 
Die Priorisierung von Informationen ist eine Frage der Sichtbarkeit, keine der theoretischen Zugänglichkeit.

\subsection{2 Äußerungsfreibeit}

Auch das Grundrecht auf freie Meinungsäußerung ist von der Aufmerksamkeitssteuerung auf Plattformen nur peripher betroffen: Als Recht auf freie Rede bzw. freie Äußerung der eigenen Meinung umfasst der Schutzbereich von Art. 5 Abs. 1 S. 1 GG zunächst die kommunikative Handlung des Äußerns, unabhängig von der Form der Äußerung (Schrift, Wort, Bild etc.). ${ }^{54}$ Auch die Wahl des zur Äußerung genutzten Kommunikationsmittels ist von der Meinungsfreiheit umfasst. ${ }^{55}$ Mit Blick auf die teils marktbeherrschenden Stellungen von Social Media-Plattformen geht die aktuelle Rechtsprechung derzeit soweit, dass Anbieter gesperrte Nutzende mit Blick auf ihre Möglichkeit zur Äußerung auf diesen Plattformen als Aspekt gesellschaftlicher Teilhabe wieder zu entsperren haben. ${ }^{56}$ Die Möglichkeit des generellen Zugangs zu diesen Plattformen erscheint so als Bestandteil der grundrechtlichen Gewährleistungen aus Art. 5 Abs. 1 GG.

Nicht umfasst vom Schutzbereich ist aber der Zugang zu Äußerungen auf der Seite der Rezipierenden - die Meinungsfreiheit gibt den Grundrechtstragenden keinen Anspruch auf die Verschaffung von Publikum. ${ }^{57}$ Vor diesem Hintergrund greift die Gewährleistung von Meinungsfreiheit im Hinblick auf algorithmische Selektionsverfahren nicht: Nicht die Äußerung an sich wird durch die Selektionsverfahren verhindert oder erschwert, sondern der Zugang des Geäußerten zu den Adressaten, wenn die Selektion zu einer Ausfilterung oder Depriorisierung führt.

54 Vgl. Bethge (2018). In: Sachs, GG, Art. 5, Rn 22 f.

55 Vgl. BVerfG Beschluss vom 10.10.1995 - 1 BvR 1476, 1980/91 und 102, 221/92, BVerfGE 93, 266 (289); Grabenwarter (87. EL März 2019). In: Maunz/Dürig, GG, Art. 5 Abs. 1, Abs. 2, Rn. 82 (m. w. N.).

56 Vgl. hier im Kontext des Sonderfalls EU-Wahlkampf: BVerfG, Beschluss der 2. Kammer des Ersten Senats vom 22.05.2019 - 1 BvQ 42/19, NJW 2019, 1935.

57 Vgl. Bethge (2018). In: Sachs, GG, Art. 5, Rn. 26a. 


\subsubsection{Objektiv-rechtliche Gewährleistung der Freiheit individueller und öfentlicher Meinungsbildung}

Die Kommunikationsverfassung des Grundgesetzes ist Gegenstand zahlreicher verfassungsgerichtlicher Konkretisierungen und Erweiterungen. ${ }^{88}$ So hat das Bundesverfassungsgericht in vielen Entscheidungen herausgearbeitet, dass Art. 5 Abs. 1 S. 2 GG einen Ausgestaltungsauftrag an den Gesetzgeber enthält, den Medienbereich positiv gesetzlich zu rahmen, um die aus Art. 5 Abs. 1 GG gelesenen Prinzipien eines Mediensystems zu gewährleisten, insbesondere die Sicherstellung der Unabhängigkeit der Medieninstitutionen vom Staat und einzelnen gesellschaftlichen Gruppen und die Gewähr einer vielfältigen Berichterstattung. ${ }^{59}$ Diese (medien-)verfassungsrechtlichen Vielfaltsgebote könnten auch Relevanz für Social Media-Anbieter entfalten, soweit diese meinungsbildungsrelevanten Informationen auf ihren Plattformen algorithmisch selektieren und so die Aufmerksamkeit bezüglich dieser Informationen auf Nutzendenseite steuern. ${ }^{60}$ Die rechtswissenschaftliche wie medienpolitische Diskussion darüber, wie positive Vielfalt auf Plattformen gemessen, operationalisiert, kontrolliert und vollzogen werden kann, wenn die vermittelten Kommunikate sowohl Individualkommunikation als auch meinungsirrelevante Inhalte und auch klassische massenmediale Kommunikation betreffen, ist umfänglich und in schneller Bewegung. ${ }^{61}$ Insoweit kann hier nicht abschließend entschieden werden, inwieweit sich der gesetzgeberische Ausgestaltungsauftrag einer positiven Vielfaltsordnung auch auf Social Media-Plattformen beziehen kann. Sicher aber ist, dass sich erweiterte Vielfaltsgebote, die sich neben publizistischen Angeboten auch auf private und halböffentliche Kommunikationen von Einzelnutzenden beziehen, außerhalb des Legislativauftrags bewegen. Für die Selektionsentscheidungen über diese Inhalte kommt eine Berührung des Schutzbereichs von Art. 5 Abs. 1 GG insoweit nicht in Betracht.

58 Vgl. nur Holznagel (2019). In: Spindler/Schuster, Recht der elektronischen Medien, RStV vor $\$ 1$ Präambel, Rn $22 \mathrm{ff}$.

59 Vgl. BVerfG Urteil vom 04.11.1986 - 1 BvF 1/84, BVerfGE 73, 118 (153 f., 161 f.); BVerfG Urteil 16.06.1981 - 1 BvL 89/78, BVerfGE 57, 295 (321 f.); BVerfG Urteil vom 27.07.1971 - 2 BvF 1/68, 2 BvR 702/68, BVerfGE 31, 314 (327).

60 Vgl. Lobigs/Neuberger (2018: S. 68-79).

61 Vgl. etwa Zimmer/Kunow (2019); Dreyer/Schulz (2019). 


\subsubsection{Objektiv-rechtliche Gewährleistung kommunikativer Chancengleichheit}

Ein für die hier bearbeitete Frage relevantes weiteres Konzept der objektivrechtlichen Seite der Medienfreiheiten aus Art. 5 Abs. 1 S. 2 GG ist das der Gewährleistung kommunikativer Chancengleichheit oder -gerechtigkeit. ${ }^{62}$ Dieser Gewährleistungsauftrag bezieht sich auf die Sachgemäßheit der Kriterien bei der Auswahl vermittelter Informationen hin zu den Rezipierenden. Damit kommt die Idee sehr nah an Teile des Betrachtungsgegenstands. Nur ist das Konzept in erster Linie ein kommunikationsfokussierter Aspekt des Äußerungsrechts und kein empfangsbezogener Anspruch auf Zugang zu bestimmten Informationen. Könnte die Plattform nachweisen, dass die algorithmische Selektion auf objektiven Kriterien beruht, erschienen die autonomie- und privatheitsbezogenen Konsequenzen dieser Entscheidungen für die Empfangenden bzw. Nicht-Empfangenden gerade nicht als Problem der kommunikativen Chancengerechtigkeit.

\subsection{Zwischenfazit: Houston, haben wir eine Grundrechtslücke?}

Das hier im Schnelldurchlauf erfolgte Mapping der grundrechtlichen Schutzbereiche und Gewährleistungsgehalte hat gezeigt, dass die klassische Schutzperspektive des Rechts auf Privatsphäre und informationelle Selbstbestimmung in erster Linie auf den Erfassungs- und Verarbeitungsschutz von personenbezogenen Daten zielt und nicht auf die aktive Aufmerksamkeitssteuerung in kommunikationsbasierten Lebensbereichen durch Dritte. ${ }^{63}$ Insbesondere die Strukturierung und Priorisierung einmal veröffentlichter Informationen sind von den derzeitigen Schutzbereichen nicht und von Gewährleistungsgehalten nur teilweise umfasst. Begrenzte Anknüpfungspunkte an bestehende grundrechtliche Schutzbereiche zeigt die Selektion zudem auf, soweit sie Relevanz für eine bestimmte Darstellung einer Person gegenüber Dritten hat. Gleichzeitig weisen algorithmische Selektionsverfahren mit Blick auf ihre Informations-, Beziehungs- und Identitätsdimensionen deutliche Konsequenzen mit Autonomie- und Integritätsbezügen auf (s. oben).

Die Zusammenschau ergibt, dass das Phänomen algorithmischer Selektion grundrechtlich derzeit kaum und wenn, dann stets nur in Teilen um-

62 Vgl. Hoffmann-Riem (2000: S. 136 f.); Kluth/Schulz (2014).

63 Diese Erkenntnis gilt übrigens auch für Art. 7, 8 GRCh; zu deren Verständnis und Entwicklungsoffenheit vgl. Marsch (2018). 
hegt ist. Es bleibt ein bislang nicht hinreichend grundrechtlich durchdrungener Bereich, in dem Dritte die kommunikative Gestaltung der informationellen und sozialen Umstände der Persönlichkeitsentwicklung und -entfaltung der Einzelnen in den Dimensionen Information, Beziehungen und Identität beeinträchtigen. Umfasst von dem bestehenden Grundrechtsrahmen sind lediglich Formen der unsachgemäßen Verarbeitung eigener Daten und - gegebenenfalls - Vielfaltseinengungen publizistischer Informationen durch Gatekeeper. Keine grundrechtliche Hilfestellung bietet das Grundgesetz dagegen bei der Abwehr algorithmischer Selektion, die die Aufmerksamkeit meiner Freundinnen und Bekannten von Informationen über mich verringert, oder die meine Aufmerksamkeit bezüglich der Informationen über bestimmte Dritte weglenkt. Es besteht hier also eine Grundrechtslücke.

\subsection{Berücksichtigung in neueren Privatheitskonzeptionen}

Die Privatheitsforschung inklusive der Betrachtung und Konzeption der normativen Gehalte von Privatheit ist den digitalen Veränderungen gegenüber nicht blind, sondern - im Gegenteil - beobachtet und begleitet diese besonders aufmerksam. So ist in den letzten zwanzig Jahren eine deutliche Veränderung zu beobachten: Das Recht auf Privatsphäre wird zunehmend zu einem Konzept eines umfassenden Rechts auf Privatheit. ${ }^{64}$ Im Rechtswissenschaftlichen wie in anderen Disziplinen sind neue Begriffe und erweiterte Konzepte von Privatheit entwickelt worden, die an die Funktionsbedingungen digitaler Netzkommunikation angepasst sind, und die auch Teile der hier herausgearbeiteten Autonomie- und Privatheitsbezüge bei algorithmischer Selektion tangieren. ${ }^{65}$

So zeigt das Konzept der, informationellen Privatheit' bei Rössler Berührungspunkte mit der oben beschriebenen Identitätsdimension und das der ,dezisionalen Privatheit ${ }^{6}$ mit der Informationsdimension auf. ${ }^{66} \mathrm{Ge}$ meinsame Bezugspunkte sind dabei vor allem Kontrollmöglichkeiten über die eigene Selbstdarstellung und die Kontrolle von Informationen, die Andere über mich publizieren. Die von Gusy propagierte Idee einer ,kommunikativen Privatheit ${ }^{\varsigma}$ betrifft vor allem die gemeinsame Aushandlung kollektiver privater Schutzräume und hat insoweit Bezüge zu der sozialen

64 Vgl. Nettesheim, VVDStRL 2011, 7 (20).

65 Vgl. umfassend etwa Koops et al., U. PA. J. INTL L. 2017, 483.

66 Vgl. Rössler (2001: S. 201). 
Kommunikation im Digitalen ${ }^{67}$ - mit dem Unterschied, dass es auf digitalen Plattformen in der Regel gerade keine Aushandlung gibt, sondern es angesichts des Machtgefälles zwischen Plattform und Nutzenden zu einer Gestaltung der Aufmerksamkeitssteuerung faktisch allein durch die Anbieter kommt. Becker und Seubert setzen mit ihrem Konzept der „kommunikativen Privatheit" ${ }^{68}$ den Fokus auf die Folgen antizipierender Verhaltensanpassungen an eine (vermeintliche) Norm und Sozialadäquanz in Anbetracht umfassender Kommunikationsüberwachung und -auswertung. Damit schaffen sie vor allem Bezüge zu den zuvor von uns beschriebenen Einflussmöglichkeiten algorithmischer Selektion auf die Wahrnehmung eines vermeintlichen Meinungsklimas und des kommunikativen Umgangs mit Andersdenkenden.

Ebenfalls Berührungspunkte zu den beschriebenen Konsequenzen weist das Privatheitskonzept der kontextuellen Integrität von Nissenbaum auf: ${ }^{69}$ Die Kontrollmöglichkeit dahingehend, dass bestimmte Informationen auf bestimmte Kontexte begrenzt bleiben, wäre auch eine Forderung, die sich mit Blick auf die Identitätsdimension der Selektionsverfahren ergibt. Dort, wo aus bewusst preisgegebenen Informationen und unbewusst hinterlassenen Datenspuren Persönlichkeits- und Interessenprofile gebildet werden und die Relevanz für die Identitätsdimension aufgezeigt wurde (s. oben), weisen die Forderungen nach dem ,Schutz vor datenbasierten Gegenbildern des Menschen' von Nettesheim und Britz bereits relevante Bezugspunkte auf: ${ }^{70}$ Deren Konzepte gehen weiter noch als Nissenbaum von der zwingenden Möglichkeit der rechtlichen Verfügung auch über Aspekte der sozialen Umwelt aus.

Zuletzt kann auch das Konzept von Eichenhöfer, der ,Privatheit als Vertrauensschutz begreift, berührt sein: ${ }^{71}$ Das Vertrauen dort bezieht sich vor allem auf die Einhaltung bestehender datenschutzrechtlicher Vorgaben, was aber bei der algorithmischen Selektion gerade nicht im Zentrum steht. Zuletzt weisen neuere Konzeptionen Berührungspunkte mit den hier beschriebenen Autonomieaspekten auf, wenn es um die durch Selektionsverfahren berührte Persönlichkeitsentwicklung und -entfaltung geht: Das Abstellen von Bock und Engeler auf die „informationelle Integrität"72

67 Vgl. Gusy, KritV 2015, 41.

68 Becker/Seubert, DuD 2016, 73.

69 Vgl. Nissenbaum (2010: S. 129, S. 186 f.).

70 Vgl. Nettesheim, VVDStRL 2011, 7 (33 f.).

71 Vgl. Eichenhofer, STAAT 2016, 41.

72 Bock/Engeler, DVBl 2016, 593 (597). 
bzw. „informationelle Unversehrtheit“73 arbeitet den Autonomiebegriff aus einer Negativ-Perspektive auf und zeigt Autonomiebeeinträchtigungen als das Fehlen von Eigensteuerungs- bzw. Selbstbestimmungsaspekten auf. In eine ähnliche Richtung gehen die Überlegungen von Jandt, die extern (mit)beeinflusste Personenbildnisse und entsprechende Fremdwahrnehmungen in ihrem Konzept der „kommunikativen Fremdbestimmung"74 eindrucksvoll beschreibt.

So hat auch die informationelle Dimension der algorithmischen Selektion bereits Berührungspunkte zu Privatheitskonzepten in der Wissenschaft: von Lewinski spricht mit Blick auf die Macht Dritter bei der Darstellung der eigenen Person von „informationeller Fremdbeschränkung“75, Roßnagel sieht so die „kommunikative Selbstbestimmung“"76, Trute die „informationelle Integrität"77 gefährdet. Soweit es um die Auswahl von zugänglich gemachten Informationen geht, erscheint der Ansatz der ,medial-rezeptiven Selbstbestimmtheit ${ }^{6}$ bei Mengden einschlägig. ${ }^{78}$ Noch weitergehend scheint hier der Begriff der „epistemischen Gewalt“79, wie ihn Elsuni in diesem Kontext geprägt hat, zu sein.

Dieser sehr kurze Überblick zeigt vor allem, dass die Privatheitsforschung offen für neue Risikodimensionen der informationellen Autonomieaspekte in der digitalen Kommunikation ist. Er zeigt aber auch, dass es Einzelaspekte sind, die die jeweiligen Konzepte berühren. Teilweise kommen die Konzepte zudem aus dem nicht-rechtswissenschaftlichen Bereich, haben also die Perspektive der oben beschriebenen (Teil-)Ausfälle der grundrechtlichen Schutzbereichszuschnitte nicht immer verinnerlicht.

\section{Recht auf autonomiewahrende Aufmerksamkeitssteuerung}

Wie oben bereits deutlich geworden ist, ist das Allgemeine Persönlichkeitsrecht - selbst als Auffanggrundrecht entwickelt - in der Konzeption des Bundesverfassungsgerichts ein umfassender Ansatz einer lückenschließenden Gewährleistung. Das Gericht begründet das nachvollziehbar:

73 Bock/Engeler, DVBl 2016, 593 (596).

74 Jandt (2015).

75 Von Lewinski (2014: S. 41 f.).

76 Roßnagel, KJ 1990, 267.

77 Trute (1998).

78 Vgl. Mengden (2018).

79 Elsuni (2018). 
Das allgemeine Persönlichkeitsrecht gewährleistet Elemente der Persönlichkeit, die nicht Gegenstand der besonderen Freiheitsgarantien des Grundgesetzes sind, diesen aber in ihrer konstituierenden Bedeutung für die Persönlichkeit nicht nachstehen [...]. Einer solchen lückenschließenden Gewährleistung bedarf es insbesondere, um neuartigen Gefährdungen zu begegnen, zu denen es im Zuge des wissenschaftlich-technischen Fortschritts und gewandelter Lebensverhältnisse kommen kann [...]. Soweit kein hinreichender Schutz vor Persönlichkeitsgefährdungen besteht, (...) trägt das allgemeine Persönlichkeitsrecht dem Schutzbedarf in seiner lückenfüllenden Funktion über seine bisher anerkannten Ausprägungen hinaus Rechnung. ${ }^{80}$

Dieser Beitrag sieht das Verständnis des grundgesetzlich verbürgten Allgemeinen Persönlichkeitsrechts in der Zusammenschau mit den autonomieund privatheitsbezogenen Risikodimensionen algorithmischer Selektionsverfahren jedenfalls als Aufforderung, sich auf die Suche nach einer möglichen Gewährleistungsausprägung zu machen. Diese sollte nicht nur die neuen, grundrechtlich nur zum Teil geschützten Aspekte dieser Verfahren abdecken, sondern auch den neueren, oben angeschnittenen Privatheitskonzepten ein integrierendes (grundgesetzliches) Zuhause bieten können.

\subsection{Aufmerksamkeitssteuerung zwischen APR und Kommunikationsfreiheiten}

Es ist deutlich geworden, dass die algorithmische Selektion als Form der Aufmerksamkeitssteuerung sowohl Privatheits- als auch Kommunikationsaspekte aufweist. Interessant ist dabei die Beobachtung, dass die kommunikationsbezogenen Konsequenzen letztendlich wieder auf Fragen der Autonomie zurückfallen - in Form einer unbeeinträchtigten Meinungsbildung, die Autonomie insbesondere in der politischen Willensbildung voraussetzt. Das steht im Widerspruch zu der derzeitigen Konzeption des Allgemeinen Persönlichkeitsrechts als Auffanggrundrecht; es scheint in seinen verästelten Spezialausprägungen zunehmend auch Vorbedingungen der erfolgreichen Ausübung eigentlich spezifischerer Freiheitsrechte abzusichern. Es spricht viel dafür, dass die spezifischen Ausprägungen des Allgemeinen Persönlichkeitsrechts gerade nicht den Charakter eines Auffanggrundrechts ,erben', sondern eigenständige und neben den spezifischen Freiheitsrechten stehende Gehalte aufweisen - ansonsten wäre 
Art. 5 Abs. 1 GG das speziellere Freiheitsrecht und eine Anwendbarkeit der persönlichkeitsrechtlichen, autonomiebezogenen Komponenten daneben nicht möglich.

Vorgeschlagen wird vor diesem Hintergrund ein integrierendes Recht auf autonomiewahrende Aufmerksamkeitssteuerung aus Art. 5 Abs. 1 i.V.m. Art. 2 Abs. 1 i.V.m. Art. 1 Abs. 1 GG. Kern dieses Grundrechts ist der Schutz gegen informationsbegrenzende, beziehungssteuernde und identitätsstiftende Fremdentscheidungen wegen ihrer Risiken für die persönlichkeitsbezogene Entwicklungs- und Entfaltungsgarantie. Dabei steht die Aufmerksamkeitssteuerung als autonomiebezogene kommunikative Handlung Dritter im Zentrum der Schutzrichtung. Die neue Ausprägung soll ein handlungsbezogener integrativer Ansatz sein, bei der die Schutzzwecke von freier Meinungsbildung und unbeeinträchtigter Persönlichkeitsentwicklung und -entfaltung verschränkt sind (s. Abb. 1). Angesichts der Verschiebung von Schutzrisiken von Seiten privater Akteure, aus deren Aktivitäten die oben beschriebenen Risiken entstehen, weist die neue Grundrechtsausprägung neben subjektiven Abwehrrechten gegen staatliche Maßnahmen auch objektiv-rechtliche Gehalte auf, die den Gesetzgeber zur Etablierung eines rechtlichen Rahmens zur Grundrechtsgewährleistung ermächtigen und ggf. verpflichten.

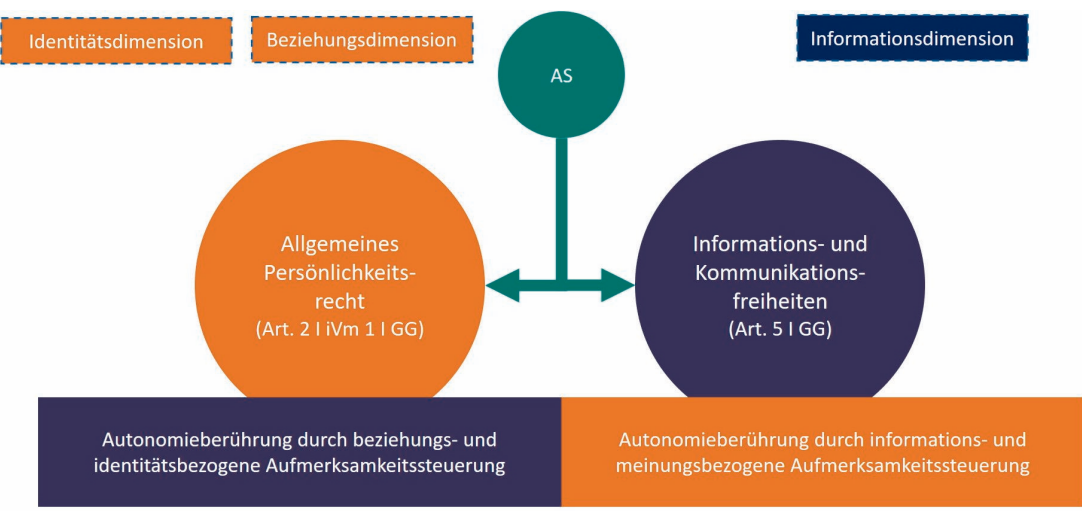

Abb. 1: Aufmerksamkeitssteuerung zwischen Allgemeinem Persönlichkeitsrecht und Kommunikationsfreiheiten (Quelle: eigene Darstellung).

\subsection{Konsequenzen für Verantwortungsgeflechte}

Ein solches Recht auf autonomiewahrende Aufmerksamkeitssteuerung kann als fehlendes Bindeglied für zukünftige Steuerungsmaßnahmen gegenüber 
großen algorithmischen Plattformen dienen. Dabei wird die Ausgestaltung der Regulierung zu berücksichtigen haben, dass die Plattformen für die Konsequenzen der algorithmischen Selektion nicht alleine verantwortlich sind. Auch wenn die Aufmerksamkeitssteuerung im Zentrum steht, sind vor allem zwei Akteure daran beteiligt: Zum einen die Anbieter, die die Systeme algorithmischer Selektion implementieren und Informationsdatenbestände vorhalten oder durchleiten; zum anderen die Nutzenden, die durch bewusste Äußerungen und beobachtbares Verhalten Input für genau jene Algorithmen geben. Zusammen mit dem dann verpflichteten Staat entstehen so gemeinsame Verantwortungsräume, die neue Formen der kooperativen Verantwortungsübernahme voraussetzen. ${ }^{81}$

Zwischen Staat und Nutzenden wird es auf die Herausarbeitung insbesondere der Gewährleistungsgehalte der neuen Grundrechtsausprägung ankommen. Im Kern wird es darum gehen müssen, wie die Absicherung der Autonomie der Nutzenden vor autonomieeinschränkender Aufmerksamkeitssteuerung rechtsfaktisch gestaltet werden kann. Wichtige Faktoren dabei werden die Erwartbarkeit des Einsatzes von Selektionsverfahren aus Sicht der Nutzenden bzw. deren Bewusstsein der Aufmerksamkeitssteuerung durch Dritte sein. Durch die bewusste Kenntnis und Akzeptanz der Aufmerksamkeitssteuerung würde die Wahrung der Autonomie der einzelnen Person hinreichend gewahrt erscheinen.

Zwischen dem Staat und den Anbietern erscheinen Verantwortungsräume weit komplexer: Da die Anbieter gleichzeitig eine große Bedeutung für ganz verschiedene Grundrechtsausübungen haben (Informations- und Meinungsfreiheit; Versammlungsfreiheit; Religionsfreiheit u. a.), weisen sie gleichzeitig positive Potenziale für Grundrechtsverwirklichung und negative für Grundrechtseinschränkungen auf. Zudem führen das staatliche Neutralitätsgebot und das Gebot der Staatsferne im Medienbereich zur Pflicht staatlicher Zurückhaltung bei der Regulierung von informationsbezogenen Intermediären. ${ }^{82}$ Vielleicht kann hier das ,Outsourcing' aufmerksamkeitssteuernder Anforderungen an gesellschaftlich rückgebundene, staats- und wirtschaftsferne Institutionen, die anstelle der Plattform entscheiden, ein guter Weg sein. ${ }^{83}$

Im Verhältnis von Anbietern und Nutzenden muss es vor allem darum gehen, Wissens- und Machtgefälle auszugleichen, um die selektionsgefährdete Autonomie zu sichern. Wissensdefizite können hier - jedenfalls zum

81 Vgl. dazu Helberger et al. (2018).

82 Vgl. Schulz/Dankert (2016: S. 52 f.).

83 Vgl. Heldt/Dreyer (im Erscheinen). 
Teil - durch Informationspflichten bzw. Transparenzgebote bezüglich der Funktionsweise ausgeglichen werden. ${ }^{84}$ Die Schaffung von Möglichkeiten der Nutzenden-Reflexion über die Gestaltungsmacht der Plattformen in Bezug auf Information, Beziehungen und Identitätsvermittlung hilft insoweit bei der Gewährleistung von Autonomie. Mit Blick auf die kommunikative und autonomieberührende Macht helfen ggf. zur Verfügung gestellte Tools, die die Gestaltungsmöglichkeit zurück in die Hände der Einzelnen legen. Solche könnten etwa die Zusicherung von kontextueller Integrität bei der Verwendung von Nutzendendaten, Nichtdiskriminierungsgarantien oder personalisierte Opt-Out-Möglichkeiten zurück zu einem neutralen, nicht personalisierten Selektionsverfahren sein.

\section{Fazit}

Die hier verfolgte Perspektive auf die unterschiedlichen Relevanzdimensionen der Funktionsweisen von Social Media-Plattformen mit Autonomie- und Privatheitsbezug ist hilfreich: Algorithmische Selektion und ihre Konsequenzen weisen Relevanz für das Informationsmanagement, für das Beziehungsmanagement, sowie für das Identitätsmanagement auf. Der Fokus der Abtastung der bestehenden grundrechtlichen Schutzbereiche erfolgte hier insoweit nicht entlang der Aushandlung von Privatheit zwischen einzelnen Personen, sondern entlang der durch Social Media-Plattformen extern vermittelten Gestaltung von Kommunikationsräumen mit Autonomiebezug: Social Media-Plattformen sind auch in diesem Sinne Intermediäre von Online-Privatheit.

Dieser Aspekt führt bestehende Schutzbereiche an Grenzen, oder fordert sie angesichts der Entgrenzung von Privatheit in mediatisierten Kontexten heraus. Der Beitrag schlägt mit Blick auf die lückenschließende Gewährleistungsfunktion des Allgemeinen Persönlichkeitsrechts eine neue Grundrechtsausprägung in Form eines Rechts auf autonomiewahrende Aufmerksamkeitssteuerung vor, die den derzeit noch lückenhaften Privatheits- und Autonomieschutz integrieren und gewährleisten kann. Die Entwicklung und Konstruktion neuer Grundrechtsausprägungen ist freilich ein langwieriger Prozess. Dieser Beitrag soll insoweit zunächst als Denkanstoß dienen. Weitere Diskussionen werden nötig sein, um die Erforderlichkeit eines neuen Schutzbereichs kritisch zu hinterfragen, ihn klar zu defi-

84 Vgl. Dreyer/Schulz (2018). 
nieren und die sich aus der Gewährleistungspflicht ergebenden Anforderungen an die praktische legislative Umsetzung zu diskutieren.

\section{Literaturverzeichnis}

Albers, Marion (2017): „Informationelle Selbstbestimmung als vielschichtiges Bündel von Rechtsbindungen und Rechtspositionen“. In: Friedewald, Michael et al. (Hrsg.): Informationelle Selbstbestimmung im digitalen Wandel. Wiesbaden: Springer Vieweg, S. 11-36.

Bazarova, Natalya et al. (2012): „Managing Impressions and Relationships on Facebook“. In: Journal of Language and Social 32 (2), S. 121-141.

Becker, Carlos (2017): „Privatheit und kommunikative Freiheit im Internet“. In: Jacob, Daniel/Thiel, Thorsten (Hrsg.): Politische Theorie und Digitalisierung. Baden-Baden: Nomos, S. 45-82.

Becker, Carlos/Seubert, Sandra (2016): „Privatheit, kommunikative Freiheit und Demokratie“. In: Datenschutz und Datensicherheit (DuD) 40 (2), S. 73-78.

Bock, Kirsten/Engeler, Malte (2016): „Die verfassungsrechtliche Wesensgehaltsgarantie als absolute Schranke im Datenschutzrecht“. In: Deutsches Verwaltungsblatt (DVBl) 131 (10), S. 593-599.

Braun, Max et al. (2018): „Perspektiven und Trends der Privatheit“. In: Friedewald, Michael (Hrsg.): Privatheit und selbstbestimmtes Leben in der digitalen Welt. Wiesbaden: Springer Vieweg, S. 221-250.

Britz, Gabriele (2007): Freie Entfaltung durch Selbstdarstellung: eine Rekonstruktion des allgemeinen Persönlichkeitsrechts aus Art. 2 I GG. Tübingen: Mohr Siebeck.

Clarke, Roger (1994): „The digital persona and its application to data surveillance“. In: The Information Society 10 (2), S. 77-92.

Clarke, Roger (2014): „Promise unfulfilled: The digital persona concept, two decades later“. In: Information Technology \& People 27, S. 182-207.

Datenethikkommission (2019): Gutachten der Datenethikkommission des Deutschen Bundestages vom 23. Oktober 2019. URL: https://www.bmi.bund.de/Shar edDocs/downloads/DE/publikationen/themen/it-digitalpolitik/gutachten-datene thikkommission.pdf?_blob=publicationFile\&v=4 [Abruf am: 23.10.2019].

Donath, Judith (2007): „Signals in social supernets“. In: Journal of Computer-Mediated Communication 13 (1), S. 231-251.

Dreyer, Stephan (2018): „Predictive Analytics aus der Perspektive von Menschenwürde und Autonomie“. In: Hoffmann-Riem, Wolfgang (Hrsg.): Big Data - Regulative Herausforderungen. Baden-Baden: Nomos, S. 135-143. 
Dreyer, Stephan/Schulz, Wolfgang (2018): Was bringt die Datenschutz-Grundverordnung für automatisierte Entscheidungssysteme? Gutachten zur neuen Datenschutz-Grundverordnung im Auftrag der Bertelsmann-Stiftung. Gütersloh: Bertelsmann-Stiftung. URL: https://www.bertelsmann-stiftung.de/fileadmin/files/B St/Publikationen/GrauePublikationen/BSt_DSGVOundADM_dt.pdf [Abruf am: 12.11.2019].

Dreyer, Stephan/Schulz, Wolfgang (2019a): Künstliche Intelligenz, Intermediäre und Öffentlichkeit. Bericht an das BAKOM. Berlin. URL: https://www.bakom.a dmin.ch/bakom/de/home/elektronischemedien/studien/einzelstudien.html [Abruf am: 12.11.2019].

Dreyer, Stephan/ Schulz, Wolfgang (2019b): Stellungnahme zum zweiten Diskussionsentwurf eines Medienstaatsvertrags der Länder vom 09. August 2019. Hamburg: Leibniz-Institut für Medienforschung | Hans-Bredow-Institut. URL: https:/ /www.hans-bredow-institut.de/uploads/media/default/cms/media/lez8f8q_HBI_ Stellungnahme2MStV.pdf [Abruf am: 12.11.2019].

Eichenhofer, Johannes (2016): „Privatheit im Internet als Vertrauensschutz“. In: Der Staat (STAAT) 55 (1), S. 41-67.

Ellison, Nicole B. et al. (2014): „Cultivating Social Resources on Social Network Sites: Facebook Relationship Maintenance Behaviors and Their Role in Social Capital Processes“. In: Journal of Computer-Mediated Communication 19 (4), S. $855-870$.

Elsuni, Sarah (2018): „Autonomie und Privatheit im Recht der informationellen Selbstbestimmung“. In: Baer, Susanne/Sacksofsky, Ute (Hrsg.): Autonomie im Recht - Geschlechtertheoretisch vermessen. Baden-Baden: Nomos, S. 119-130.

Gusy, Christoph (2015): „Privatheit und Demokratie“. In: Kritische Vierjahreszeitschrift für Gesetzgebung und Rechtswissenschaft (KritV) 98 (4), S. 430-461.

Helberger, Natali et al. (2018): „Governing online platforms: From contested to cooperative responsibility“. In: The Information Society 34 (1), S. 1-14.

Heldt, Amélie/Dreyer, Stephan (im Erscheinen): „Competent third parties and content moderation on platforms: Potentials of independent decision-making bodies from a governance structure perspective ".

Hoffmann-Riem, Wolfgang (2000): Regulierung der dualen Rundfunkordnung. Baden-Baden: Nomos.

Hoffmann-Riem, Wolfgang (2016): Innovation und Recht - Recht und Innovation. Recht im Ensemble seiner Kontexte. Tübingen: Mohr Siebeck.

Hoffmann-Riem, Wolfgang (2018) (Hrsg.): Big Data - Regulative Herausforderungen. Baden-Baden: Nomos.

Hornung, Gerrit (2015): Grundrechtsinnovationen. Tübingen: Mohr Siebeck.

Jandt, Silke (2015): „Der Januskopf des Internet - Zur Ambivalenz von Selbst- und Fremdbestimmung im Internet“. In: Hruschka, Joachim/Joerden, Jan C. (Hrsg.): Jahrbuch für Recht und Ethik. Berlin: Duncker \& Humblot, S. 53-74.

Katzenbach, Christian (2018): Die Regeln digitaler Kommunikation. Governance zwischen Norm, Diskurs und Technik. Wiesbaden: Springer VS. 
Kluth, Winfried/Schulz, Wolfgang (2014): Konvergenz und regulatorische Folgen. Gutachten im Auftrag der Rundfunkkommission der Länder. Oktober 2014. URL: http://epub.sub.uni-hamburg.de/epub/volltexte/2017/63834/pdf/30Konver genz.pdf [Abruf am: 12.11.2019].

Koops, Bert-Jaap et al. (2017): „A Typology of Privacy“. In: University of Pennsylvania Journal of International Law (U. PA. J. INTL L.) 38 (2), S. 483-575.

Kosinski, Michal/Wang, Yilun (2018): „Deep neural networks are more accurate than humans at detecting sexual orientation from facial images“. In: Journal of Personality and Social Psychology 114 (2), S. 246-257.

Krämer, Nicole C./Haferkamp, Nina (2011): „Online self-presentation: Balancing privacy concerns and impression construction on social networking sites“. In: Trepte, Sabine/Reinecke, Leonard (Hrsg.): Privacy online: Perspectives on privacy and self-disclosure in the social web. Berlin u. a.: Springer, S. 127-142.

Ladeur, Karl-Heinz (2009): „Das Recht auf informationelle Selbstbestimmung: Eine juristische Fehlkonstruktion?" In: Die Öffentliche Verwaltung (DÖV) 62 (2), S. 45-55.

von Lewinski, Kai (2014): Die Matrix des Datenschutzes: Besichtigung und Ordnung eines Begriffsfeldes. Tübingen: Mohr Siebeck.

Lobigs, Frank/Neuberger, Christoph (2018): Meinungsmacht im Internet und die Digitalstrategien von Medienunternehmen. Schriftenreihe der Landesmedienanstalten. Berlin: Vistas.

Marsch, Nikolaus (2018): Das europäische Datenschutzgrundrecht: Grundlagen, Dimensionen, Verflechtungen. Tübingen: Mohr Siebeck.

Maunz, Theodor/Dürig, Günter (2019): Grundgesetz. Kommentar. 87. Auflage, März 2019. München: C.H. Beck.

Mengden, Martin (2018): Zugangsfreiheit und Aufmerksamkeitsregulierung: zur Reichweite des Gebots der Gewährleistung freier Meinungsbildung am Beispiel algorithmengestützter Zugangsdienste im Internet. Tübingen: Mohr Siebeck.

Mühlichen, Andreas (2018): Privatheit im Zeitalter vernetzter Systeme. Opladen u. a.: Verlag Barbara Budrich.

Nagenborg, Michael (2005): Das Private unter den Rahmenbedingungen der luKTechnologie. Wiesbaden: VS Verlag.

Napoli, Philip M. (2014): „Automated Media. An Institutional Theory Perspective on Algorithmic Media Production and Consumption“. In: Communication Theory 24 (3), S. 340-360.

Nassehi, Armin (2019): „Die Zurichtung des Privaten. Gibt es analoge Privatheit in einer digitalen Welt?“ In: Stempfhuber, Martin/Wagner, Elke (Hrsg.): Praktiken der Überwachten. Wiesbaden: Springer VS, S. 63-77.

Nettesheim, Martin (2011): „Grundrechtsschutz der Privatheit“. In: Veröffentlichungen der Vereinigung der Deutschen Staatsrechstlehrer (VVDStRL) 70, S. 749.

Nissenbaum, Helen Fay (2010): Privacy in context: technology, policy, and the integrity of social life. Stanford: Stanford Law Books. 
Pasquale, Frank A./Cockfield, Arthur J. (2018): „Beyond Instrumentalism: A Substantivist Perspective on Law, Technology, and the Digital Persona“. In: Michigan State Law Review (4), S. 821-868.

Petersen, Thomas et al. (2017): „Der Einfluss der Nutzung sozialer Netzwerke auf die Wahrnehmung des Meinungsklimas“. In: Jandura, Olaf et al. (Hrsg.): Zwischen Integration und Diversifikation. Wiesbaden: Springer VS, S. 229-244.

Rössler, Beate (2001): Der Wert des Privaten. Frankfurt am Main: Suhrkamp.

Rössler, Beate (2018): „Privatheit, Autonomie, Recht“. In: Baer, Susanne/Sacksofsky, Ute (Hrsg.): Autonomie im Recht - Geschlechtertheoretisch vermessen. Baden-Baden: Nomos, S. 93-118.

Roßnagel, Alexander (1990): „Das Recht auf (tele-)kommunikative Selbstbestimmung“. In: Kritische Justiz (KJ) 23 (3), S. 267-289.

Sachs, Michael (2018) (Hrsg.): Grundgesetz. 8. Auflage. München: C.H. Beck.

Schmidt, Jan-Hinrik (2006): „Social Software: Onlinegestütztes Informations-, Identitäts- und Beziehungsmanagement“. In: Forschungsjournal Soziale Bewegungen 19 (2), S. 37-47.

Schmidt, Jan-Hinrik (2017): Das neue Netz: Merkmale, Praktiken und Folgen des Web 2.0. Köln: Herbert von Halem.

Schmidt, Jan-Hinrik et al. (2018): „Wie können Empfehlungssysteme zur Vielfalt von Medieninhalten beitragen?" In: Media Perspektiven 11, S. 523-531.

Schoch, Friedrich (2011): „Der verfassungsrechtliche Schutz des Fernmeldegeheimnisses (Art. 10 GG)“. In: Juristische Ausbildung (Jura) 33 (3), S. 194-204.

Schulz, Wolfgang/Dankert, Kevin (2016): Die Macht der Informationsintermediäre: Erscheinungsformen, Strukturen und Regulierungsoptionen. Berlin: FES.

Smith, Brendan (2017): „Data Shadows and Digital Personae: Alienated Figures of User Identity in Social Media“. In: The iJournal 3 (1). URL: https://theijournal.ca/index.php/ijournal/article/view/28892 [Abruf am: 12.11.2019]

Solove, Daniel J. (2004): The Digital Person: Technology and Privacy in the Information Age. New York: New York University Press.

Spindler, Gerald/Schuster, Fabian (2019): Recht der elektronischen Medien. 4. Auflage. München: C.H. Beck.

Staben, Julian (2016): Der Abschreckungseffekt auf die Grundrechtsausübung. Tübingen: Mohr Siebeck.

Stark, Birgit et al. (2017): Ganz meine Meinung? Informationsintermediäre und Meinungsbildung - Eine Mehrmethodenstudie am Beispiel von Facebook. LfMDokumentation Band 55. Düsseldorf: LfM.

Steinmüller, Wilhelm et al. (1971): Grundfragen des Datenschutzes. Gutachten im Auftrag des Bundesministeriums des Innern. BT-Drs. VI/3826, S. 7-161.

Stempfhuber, Martin (2019): „Neue Trends im Strukturwandel der Privatheit“. In: Stempfhuber, Martin/Wagner, Elke (Hrsg.): Praktiken der Überwachten. Wiesbaden: Springer VS, S. 157-179. 
Thelwall, Mike/Wilkinson, David (2010): „Public dialogs in social network sites: What is their purpose?" In: Journal of the American Society for Information Science and Technology 61 (2), S. 392-404.

Tong, Stephanie Tom/Walther, Joseph B. (2011): „Relational maintenance and CMC“. In: Wright, Kevin B./Webb, Lynne M. (Hrsg.): Computer-mediated communication in personal relationships. New York: Peter Lang, S. 98-118.

Trepte, Sabine (2016): „Die Zukunft der informationellen Selbstbestimmung Kontrolle oder Kommunikation?" In: Stiftung Datenschutz (Hrsg.): Die Zukunft der informationellen Selbstbestimmung. Berlin: Erich Schmidt, S. 159170.

Trute, Hans-Heinrich (1998): „Öffentlich-rechtliche Rahmenbedingungen einer Informationsordnung“. In: Veröffentlichung der Vereinigung der Deutschen Staatsrechtslehrer (VVDStRL) 57, S. 216-268.

Tufekci, Zeynep (2008): „Grooming, gossip, Facebook, and MySpace“. In: Information, Communication \& Society 11 (4), S. 544-564.

Zerfaß, Ansgar et al. (2008) (Hrsg.): Kommunikation, Partizipation und Wirkungen im Social Web: Grundlagen und Methoden. Von der Gesellschaft zum Individuum. Köln: Herbert von Halem.

Zimmer, Anja/Kunow, Kristian (2019): „Intermediäre und Meinungsbildung“. In: die Medienanstalten (Hrsg.): Vielfaltsbericht 2019 der Landesmedienanstalten. Berlin: die Medienanstalten, S. 46-59. 
